\title{
AUTOMORPHISMS OF STRUCTURAL MATRIX ALGEBRAS
}

\author{
Mustafa AkKurt, Emira AkKurt And George P. BARKer
}

Abstract. The topic considered in this paper falls under the general heading of automorphisms of structural matrix algebras. Herein we wish to give an answer to an open question given in [4]. We also would like to reprove of Theorem A and Theorem C in [6], a version of the principal results, by using the structure of the algebra in the block upper triangular case.

Mathematics subject classification (2010): 15A30, 16S50, 08A35.

Keywords and phrases: structural matrix algebras, block triangular matrices, automorphisms.

\section{REFERENCES}

[1] A. A. Albert, Modern Higher Algebra, The University of Chicago Science Series, 1937.

[2] M. AKKURT, G. P. BARKER AND M. WILD, Structural matrix algebra and their lattices of invariant subspaces, Linear Algebra and its Appl. 394, (2005), pp. 25-38.

[3] G. P. BARKER AND T. P. KeZLAN, The automorphism group of matrix algebra, in Current Trends in Matrix Theory (F. Uhlig and R. G. Crone, Eds), North-Holland, New York, (1987).

[4] G. P. BARKER, Automorphism group of algebras of triangular matrices, Linear Algebra and its Appl. 121, (1989), 207-215.

[5] G. P. BARKeR AND T. P. Kezlan, Automorphism of algebras of upper triangular matrices, Arch. Math. 55, (1990), 38-43.

[6] S. P. Coelho, The automorphism group of structural matrix algebra, Linear Algebra and its Appl. 195, (1993), 35-58.

[7] D. R. FARENICK, Algebras of Linear Transformations, Unversitext Springer, 2001.

[8] I. M. Is AACS, Automorphism of matrix algebras over commutative rings, Linear Algebra and its Appl. 31, (1980), 215-231.

[9] S. JondRUP, Automorphisms of upper triangular matrix rings, Arch. Math. (Basel), 49, (1987), 497502.

[10] T. J. LAfFEY, A structure theorem for some matrix algebras, Linear Algebra and its Appl. 162-164, (1992), 205-215. 